\title{
Diseño y evaluación de una aplicación para Educación Física de orientación deportiva en el Medio Natural
}

\section{Design and evaluation for Physical Education of a sports orienteering application in the Natural Environment}

\author{
Remigio Pérez Rabadán \\ IES Cura Valera de Huércal-Overa (España) \\ remigio.perezr@um.es
}

Recibido: $17 / 06 / 2020$

Aceptado: 06/04/2021

Publicado: 01/06/2021

\section{RESUMEN}

Se trata de una propuesta de innovación que pretende ser realizada en el contexto de las actividades en el medio natural en relación con las tecnologías de la información y comunicación a través de la materia de Educación Física en un centro educativo de Educación Secundaria. Se pretende poder innovar en nuestro contexto educativo con la incorporación de las nuevas tecnologías y poder comprobar los resultados, siendo así el centro de nuestro trabajo el diseño y evaluación de una aplicación que creemos mejora la experiencia de aprendizaje de los estudiantes. La intervención ha consistido, por tanto, en el desarrollo e implementación de una aplicación móvil (Orienta Educación Deportiva) basándonos para ello en el análisis de los criterios de diseño y usabilidad de varias aplicaciones relacionadas con la materia, que propone caminos interactivos y actualizados en el proceso educativo, con el fin de mejorar y enriquecer el proceso de Enseñanza-Aprendizaje del alumnado mediante el uso de dispositivos digitales.

En consecuencia, cien alumnos han usado y evaluado esta aplicación durante el curso escolar 20192020. Para la evaluación, han cumplimentado un cuestionario sobre su usabilidad y experiencia en el proceso de Enseñanza-Aprendizaje, así como expresado su opinión en relación al uso de la aplicación en orientación deportiva. Tras el análisis de los resultados obtenidos se puede observar que los alumnos valoran la aplicación de forma muy positiva en referencia a las características de la misma con valores de conformidad por encima del $85 \%$, mostrando también un grado de adquisición de nuevos contenidos en su experiencia de aprendizaje con valores del 87 \%. La Educación Física está llamada a seguir procesos de inmersión digital, asociados al uso de tecnología, especialmente la móvil (Díaz,2019). Por lo que con la implantación de esta propuesta de innovación se intenta fomentar el uso de las nuevas tecnologías.

\section{PALABRAS CLAVE}

Innovación; Actividad Física; Aplicación móvil; Evaluación; Educación Secundaria

\section{ABSTRACT}

This is an innovation proposal that aims to be carried out in the context of activities in the natural environment in relation to information and communication technologies through the subject of Physical Education in a Secondary High School. The aim is to be able to innovate in our educational context with the incorporation of new technologies and to be able to verify the results, thus being the center of our work the design and evaluation of an application that we believe improves the learning experience of students. The intervention has consisted, therefore, of the development and implementation of a mobile application (Orienta Educación Deportiva) based on the analysis of the design criteria and usability of various applications related to the subject, which proposes interactive and updated paths in the educational process, in order to improve and enrich the teaching-learning process of the students through the use of digital devices.

Consequently, one hundred students have used and evaluated this application during the 2019-2020 school year. For the evaluation, they have completed a questionnaire about their usability and experience 
in the teaching-learning process, as well as expressed their opinion in relation to the use of the application in orientation. After analyzing the results obtained, it can be seen that the students value the application in a very positive way in reference to its characteristics, with conformity values above $85 \%$, also demonstrating a degree of acquisition of new contents in their learning experience. with values of $87 \%$. The Physical Education is called to follow processes of digital immersion, associated with the use of technology, especially mobile (Díaz, 2019). Therefore, with the implementation of this innovation proposal, an attempt is made to promote the use of new technologies.

\section{KEYWORDS}

Innovation; Physical Activity; Mobile Application; Evaluation; Secondary education

\section{CITA RECOMENDADA}

Pérez, R. (2021). Diseño y evaluación de una aplicación para Educación Física de orientación deportiva en el Medio Natural. Revista Interuniversitaria de Investigación en Tecnología Educativa, 10, 43-61. https://doi.org/10.6018/riite.432941

\section{Principales aportaciones del artículo y futuras líneas de investigación:}

- Aporta una visión de la actualidad, donde la experiencia de aprendizaje entre distintos espacios educativos puede ser apoyada a través de las Nuevas Tecnologías, mediante una aplicación educativa.

- El uso de las herramientas digitales mediante una aplicación gratuita en los procesos didácticos que permite innovar en los contenidos y en la forma de enseñar la materia, aportando numerosos beneficios para el alumnado en su proceso de Enseñanza-Aprendizaje.

- Se pueden desarrollar mejoras de la aplicación y similares en función a los criterios de diseño y usabilidad con una muestra más amplia y con más margen de tiempo para comparar y modificar.

\section{INTRODUCCIÓN}

El marco que sustenta este trabajo es una propuesta de innovación apoyada en el uso de las Tecnologías de la Información y Comunicación (TIC), basada en el diseño de recursos y materiales digitales para la formación, como es el diseño de una aplicación (app) de Orientación Deportiva, y los efectos producidos en el proceso de Enseñanza-Aprendizaje (E-A). Relacionado con esto, este es un proyecto orientado a la incorporación de las TIC en la enseñanza presencial, en el marco de la asignatura de Educación Física (E.F). en la Unidad Didáctica (U.D) de Orientación Deportiva.

Así, algunos de los efectos de los avances tecnológicos en el proceso de E-A son las posibilidades de las TIC para la enseñanza y el aprendizaje, el efecto del uso de las TIC en el aprendizaje, el análisis del uso en el sistema educativo, la competencia digital y el uso de recursos TIC (Prendes \& González, 2017). Díaz (2018), sugiere que más que un problema tecnológico en el sistema educativo existe un problema de formación en el profesorado y alumnado, de uso y señala que el único inconveniente que plantea un dispositivo móvil es no saber utilizarlo de manera idónea.

El uso de aplicaciones móviles en la práctica deportiva se ha convertido en algo habitual hoy en día (Aznar et al., 2019). Los dispositivos digitales están totalmente integrados en nuestra sociedad, por lo que están llamados a cambiar la forma de enseñar y de aprender (Díaz, 2019). Los dispositivos móviles poseen utilidades innegables: multifuncionalidad, portabilidad, compatibilidad y menor coste, pero no todo son ventajas (Lavay et al., 2015). Para Aubusson et al., (2016) o Traxler (2016), su uso está condicionado por determinadas creencias del 
profesorado sobre la vulnerabilidad de las redes, la privacidad de los datos y por actitudes negativas sobre la validez y la fiabilidad de las apps.

Por tanto, dentro de la materia de E.F, la experiencia de aprendizaje puede ser apoyada a través de las TIC con la relación existente entre espacios físicos y virtuales gracias a los dispositivos móviles con herramientas como códigos QR y geo posición (Vázquez-Cano \& Sevillano, 2015). Las diferentes aplicaciones disponibles para el desarrollo de la actividad física monitorizada o como interrelación entre diferentes deportistas son aplicables a las actividades físico-recreativas en medio natural tanto a nivel deportivo, turístico y/o educativo (Generelo, 2010). Así, la innovación y la investigación son primordiales para aprovechar el inmenso potencial de mejora y trasformación educativa que tiene la tecnología móvil en la EF (Díaz, 2019).

En la materia de E.F. son muchas las investigaciones que estudian el potencial educativo de integrar las nuevas tecnologías, obteniendo como resultados el trabajo autónomo, recursos innovadores, cooperación, relaciones interpersonales, resolución de retos, reflexión y aprendizaje constructivo (Prat et al., 2013). La práctica de Actividades Físicas en el Medio Natural (AFMN) está en pleno auge en la actualidad, siendo nota de esto que cada vez son más los investigadores que deciden publicar e investigar en este campo, como por ejemplo en el último año (Escaravajal \& Baena, 2019). Para el profesorado, el análisis de la usabilidad tecnológica, no es suficiente garantía de calidad, también se necesita valorar la usabilidad pedagógica (Turpo, 2018). Los motivos por los que la actividad con TIC presenta un atractivo formativo añadido a la orientación deportiva en el medio natural se presentan a continuación (véase la tabla 1).

Tabla 1 Motivos de utilización de las TIC en Orientación Deportiva (Rodríguez et al. 2017)

\begin{tabular}{|c|c|}
\hline Actividad & Descripción \\
\hline No competitiva & $\begin{array}{c}\text { Facilita la cooperación entre los alumnos sin necesidad del componente } \\
\text { competitivo }\end{array}$ \\
\hline No habitual & $\begin{array}{l}\text { La actividad en sí misma es una incógnita y reto para el estudiante que } \\
\text { propicia motivación e implicación en la misma }\end{array}$ \\
\hline Cooperativa & $\begin{array}{c}\text { Trabajo en equipo para conseguir el éxito u objetivo planteado ya sea } \\
\text { interpretando el medio, localizando balizas... }\end{array}$ \\
\hline $\begin{array}{l}\text { Con posibilidad de } \\
\text { éxito }\end{array}$ & $\begin{array}{c}\text { Éxito al alcance de todos sin importar las características y capacidades de } \\
\text { cada alumno, así como las habilidades previas }\end{array}$ \\
\hline Sin experiencia previa & Todos los participantes parten del mismo punto de partida \\
\hline Accesible & $\begin{array}{c}\text { Los dispositivos móviles están al alcance de todos con aplicaciones a coste } \\
\text { cero. }\end{array}$ \\
\hline Inclusiva & $\begin{array}{c}\text { Permite variedad de participantes sin importar edad, nivel o que presenten } \\
\text { algún tipo de diversidad funcional. }\end{array}$ \\
\hline
\end{tabular}

En definitiva, este trabajo de innovación trata de describir tanto el proceso de diseño de la aplicación, a partir de la justificación sustentada en el análisis de experiencias, investigaciones y de otras aplicaciones, como el proceso de evaluación recogiendo datos empíricos de la experiencia de los estudiantes que la han utilizado en contextos reales de tareas de orientación. La aplicación diseñada pretende contribuir a construir una experiencia educativa más emocionante y enriquecedora para nuestros estudiantes. Por tanto, la actividad está vinculada de forma directa a los estándares determinados en el currículum oficial de la asignatura y contribuye a obtener los resultados de aprendizaje perseguidos.

\subsection{Problema o situación que se pretende mejorar de la realidad educativa}

La E.F. en esta nueva era demanda nuevos conocimientos, competencias y habilidades. No podemos decidir si queremos o no usar la tecnología: debemos decidir cómo la vamos a incorporar de forma eficaz a los procesos de Enseñanza-Aprendizaje dependiendo del contexto educativo (Aznar et al., 2019). La introducción de las TIC, y más concretamente de las aplicaciones de Orientación Deportiva con la utilización de códigos QR en la esfera educativa, es mayor día a día debido a la potencialidad que esta herramienta representa (Barahona, 2012). 
Como base para fundamentar esta situación a mejorar se tomaron como referentes varios estudios en relación con diferentes modalidades deportivas y el uso de las TIC (véase tabla 2).

Tabla 2 Propuestas relacionadas con el uso de las TIC y la mejora de los procesos de E-A

\begin{tabular}{|c|c|c|c|}
\hline DEPORTE & AUTORES & ESTUDIO & DESCRIPCIÓN \\
\hline Balonmano & $\begin{array}{l}\text { Abril et al. } \\
\text { (2015) }\end{array}$ & $\begin{array}{c}\text { "Aplicación de las TIC como } \\
\text { herramienta didáctica para la } \\
\text { Enseñanza - aprendizaje del } \\
\text { balonmano" }\end{array}$ & $\begin{array}{l}\text { Los resultados muestran la } \\
\text { evolución de los estudiantes y el } \\
\text { manejo conceptual de la disciplina } \\
\text { del balonmano con el uso de TIC. }\end{array}$ \\
\hline Pádel & $\begin{array}{l}\text { Sánchez } \\
\text { Alcaraz } \\
(2015)\end{array}$ & $\begin{array}{l}\text { "La utilización de videos } \\
\text { didácticos en la enseñanza- } \\
\text { aprendizaje de los golpes de } \\
\text { pádel en estudiantes" }\end{array}$ & $\begin{array}{l}\text { La utilización de herramientas } \\
\text { digitales en los procesos de E-A en } \\
\text { E.F ha sido valorada de forma } \\
\text { positiva hacia su aprendizaje } \\
\text { despertando mayor interés. }\end{array}$ \\
\hline Voleibol & $\begin{array}{l}\text { Reyes } \\
(2019)\end{array}$ & $\begin{array}{l}\text { "Apropiación conceptual } \\
\text { fundamentos técnicos del } \\
\text { voleibol en ambientes de } \\
\text { aprendizaje: con mediación } \\
\text { de las TIC frente al } \\
\text { aprendizaje tradicional" }\end{array}$ & $\begin{array}{l}\text { Las TIC en E.F permiten que el } \\
\text { estudiante pueda tener mejores } \\
\text { resultados en su desempeño } \\
\text { académico pues ofrecen múltiples } \\
\text { beneficios que no pueden aportar el } \\
\text { modelo tradicional de aprendizaje. }\end{array}$ \\
\hline Fútbol & $\begin{array}{l}\text { Ortega et al. } \\
\qquad(2008)\end{array}$ & $\begin{array}{c}\text { "Nuevas Tecnologías } \\
\text { aplicadas a la cuantificación } \\
\text { en el fútbol" }\end{array}$ & $\begin{array}{l}\text { Las TIC favorecen al estudio de } \\
\text { diferentes parámetros del futbolista } \\
\text { dentro del terreno de juego } \\
\text { físicamente, tácticamente, etc.). }\end{array}$ \\
\hline $\begin{array}{l}\text { Orientación } \\
\text { Deportiva }\end{array}$ & $\begin{array}{l}\text { Gallego et } \\
\text { al. (2017) }\end{array}$ & $\begin{array}{c}\text { "La orientación en el medio } \\
\text { natural: aprendizaje ubicuo } \\
\text { mediante el uso de } \\
\text { tecnología" }\end{array}$ & $\begin{array}{c}\text { Analizó cómo el aprendizaje ubicuo } \\
\text { a través del uso de herramientas TIC } \\
\text { (Códigos QR, RA, etc.), apoya el } \\
\text { aprendizaje de contenidos de } \\
\text { orientación. }\end{array}$ \\
\hline $\begin{array}{l}\text { Actividad } \\
\text { Física Medio } \\
\text { Natural }\end{array}$ & $\begin{array}{c}\text { Alcaraz et al. } \\
(2018)\end{array}$ & $\begin{array}{l}\text { "El gran juego salvando a } \\
\text { gea: gamificación y nuevas } \\
\text { tecnologías en actividades } \\
\text { físicas en medio natural" }\end{array}$ & $\begin{array}{l}\text { La AFMN facilita la asunción del rol } \\
\text { asignado motivando e implicando } \\
\text { más al alumno, que hace que trabaje } \\
\text { en equipo. Las TIC son una } \\
\text { herramienta didáctica que hace } \\
\text { atractiva, participativa y motivante el } \\
\text { proceso de E-A. }\end{array}$ \\
\hline
\end{tabular}

Tras observar la realización y resultado de esta U.D en años anteriores realizada de forma tradicional ,el análisis de los estudios entre el uso de las TIC y modelo tradicional (tabla 2) y la prueba piloto realizada a un grupo de estudiantes con la app antes de la propuesta de innovación, nos lleva a deducir que los problemas detectados en nuestra realidad concreta serían que el aprendizaje significativo de los contenidos de la materia no se alcanza en su plenitud, ya que la mayoría no supera los estándares de aprendizaje valorados en la U.D de Orientación Deportiva; y también que el interés y motivación del alumnado no es el deseado. La predisposición hacia la realización de la UD no es la adecuada. Por tanto, la situación a mejorar está relacionada con la adquisición de conocimientos de Orientación Deportiva en nuestros alumnos a través de la implementación y desarrollo de una Aplicación de Orientación Deportiva en Educación Secundaria, analizando la experiencia de aprendizaje de alumnos de Secundaria a través del diseño de una aplicación para tal efecto.

Así, se pretende valorar la posibilidad de que, a través de los dispositivos móviles con la utilización de una aplicación (donde en un mismo dispositivo se encuentre mapa, balizas, preguntas de contenidos de orientación,...) mediante herramientas como códigos QR puedan mejorar las experiencias de aprendizaje con la adquisición de conocimientos más constructivos. 


\subsection{Aplicaciones de orientación deportiva}

En la clasificación de Escaravajal \& Baena (2019) respecto a aplicaciones relacionadas con Orientación Deportiva (véase tabla 3), se han seleccionado aquellas directamente relacionadas con nuestra propuesta que comprenden características básicas en el marco escolar: de carácter y acceso gratuito, con idioma en castellano y/o inglés, con herramientas como códigos QR y geo localizadores, con sencillez y claridad en la usabilidad de la aplicación.

Tabla 3. Aplicaciones relacionadas con orientación deportiva (Escaravajal \& Baena, 2019, pp. 33-36)

\begin{tabular}{|c|c|c|}
\hline $\begin{array}{l}\text { APLICACIÓN } \\
\text { PLATAFORMA } \\
\text { DISPONIBLE }\end{array}$ & $\begin{array}{l}\text { IDIOMA } \\
\text { ACCESO }\end{array}$ & DESCRIPCIÓN \\
\hline $\begin{array}{c}\text { IORIENTEERING } \\
\text { Aplicación móvil } \\
\text { http://www.iorienteering.com/ }\end{array}$ & $\begin{array}{l}\text { Inglés/ } \\
\text { Gratuito }\end{array}$ & $\begin{array}{l}\text { El alumno tiene que acudir a los puntos } \\
\text { indicados y escanear el código QR, así } \\
\text { confirmará su paso por los controles. }\end{array}$ \\
\hline $\begin{array}{l}\text { DIB ORIENTEERING DIBBER } \\
\text { Aplicación móvil } \\
\text { http://www.appindesign.com/ dib/ }\end{array}$ & $\begin{array}{l}\text { Inglés/ } \\
\text { Gratuito }\end{array}$ & $\begin{array}{l}\text { Permite cronometrar las carreras de } \\
\text { orientación a través de códigos QR. El } \\
\text { cronometrador dispondrá de otra app. }\end{array}$ \\
\hline $\begin{array}{c}\text { SPORTEERING } \\
\text { Aplicación móvil } \\
\text { http://www.sporteering.com/ }\end{array}$ & $\begin{array}{l}\text { Español e } \\
\text { inglés/ } \\
\text { Gratuito }\end{array}$ & $\begin{array}{c}\text { Navegar por una serie de controles y } \\
\text { acumular puntos a medida que los alcanzas, } \\
\text { con códigos QR. }\end{array}$ \\
\hline $\begin{array}{c}\text { MOBO } \\
\text { Aplicación móvil } \\
\text { http://mobo.osport.ee/ }\end{array}$ & $\begin{array}{l}\text { Español, } \\
\text { inglés y otros } \\
\text { idiomas/ } \\
\text { Gratuito }\end{array}$ & $\begin{array}{l}\text { Aplicación innovadora de } 3 \text { en } 1 \text { para los } \\
\text { orientadores: mapa, brújula y sistema de } \\
\text { registro de paso por los controles. }\end{array}$ \\
\hline $\begin{array}{c}\text { O-TRACK } \\
\text { Aplicación móvil } \\
\text { https://o-track.dk/da/about }\end{array}$ & $\begin{array}{l}\text { Inglés/ } \\
\text { Gratuito }\end{array}$ & $\begin{array}{c}\text { Utiliza GPS del teléfono móvil para rastrear } \\
\text { su carrera, subir su pista a la pista-O, luego } \\
\text { volver y rastrear. }\end{array}$ \\
\hline $\begin{array}{c}\text { O-MOPSI } \\
\text { Aplicación móvil } \\
\text { http://cs.uef.fi/o-mopsi/ }\end{array}$ & $\begin{array}{l}\text { Inglés/ } \\
\text { Gratuito }\end{array}$ & $\begin{array}{c}\text { Juego de orientación al aire libre. Encontrar } \\
\text { lugares del mundo real utilizando el teléfono } \\
\text { y su GPS. }\end{array}$ \\
\hline $\begin{array}{c}\text { ORIENTGAME } \\
\text { Aplicación móvil } \\
\text { https://www.orientgame.com/ }\end{array}$ & $\begin{array}{l}\text { Inglés/ } \\
\text { Gratuito }\end{array}$ & $\begin{array}{c}\text { Orientación sin brújula para el medio urbano. } \\
\text { Utiliza la propia ubicación del dispositivo y el } \\
\text { sistema de mapas. }\end{array}$ \\
\hline $\begin{array}{l}\text { ORIENTEERINGWAY } \\
\text { Aplicación móvil }\end{array}$ & $\begin{array}{l}\text { Inglés/ } \\
\text { Gratuito }\end{array}$ & $\begin{array}{l}\text { Juego de orientación en tiempo real. } \\
\text { Navegar a través de puntos de control en la } \\
\text { región } 2 \mathrm{D} \text { en el mapa y la brújula. }\end{array}$ \\
\hline
\end{tabular}

\subsection{Revisión de propuestas, experiencias e investigaciones educativas}

Dentro del ámbito educativo son cada vez más los estudios desarrollados sobre la influencia de las TIC en el proceso de E-A del alumnado, y más concretamente en herramientas digitales relacionadas con las AFMN. El diseño utilizado para esta propuesta de innovación ha sido la revisión documental descriptiva de la bibliografía científica relacionada con las apps tecnológicas, el deporte de orientación y la E.F. Se han elegido aquellas propuestas, experiencias e investigaciones consideradas más relevantes en relación a la propuesta (véase tabla 4, 5 y 6 ).

Para la selección de las propuestas educativas (véase tabla 4), los términos de búsqueda han sido: propuestas novedosas y recientes, donde se han tomado como referencia que se hayan realizado con alumnos de Secundaria principalmente, utilizando sobre todo geo localizadores y códigos QR ya que la propuesta de innovación se basa en estas dos herramientas. 
Tabla 4. Revisión de propuestas educativas

\begin{tabular}{|c|c|c|c|}
\hline Autoría & $\begin{array}{l}\text { Herramientas } \\
\text { TIC }\end{array}$ & $\begin{array}{l}\text { Alumnado } \\
\text { participante }\end{array}$ & Descripción \\
\hline $\begin{array}{l}\text { Alcaraz et al. } \\
\quad(2018)\end{array}$ & Códigos QR & $\begin{array}{l}\text { Secundaria y } \\
\text { Educación } \\
\text { Superior }\end{array}$ & $\begin{array}{c}\text { Geolocalización de las diferentes } \\
\text { postas, dentro de un juego } \\
\text { grupal. }\end{array}$ \\
\hline $\begin{array}{l}\text { Fernández et al. } \\
\qquad(2017)\end{array}$ & $\begin{array}{l}\text { Geocaching e } \\
\text { iOrienteering }\end{array}$ & $\begin{array}{l}\text { Secundaria y } \\
\text { Bachillerato }\end{array}$ & $\begin{array}{l}\text { Utilización de varias apps } \\
\text { móviles relacionadas con las } \\
\text { actividades en la naturaleza }\end{array}$ \\
\hline $\begin{array}{l}\text { Gallego et al. } \\
\quad(2017)\end{array}$ & $\begin{array}{l}\text { iOrienteering, } \\
\text { Geocaching, Wikiloc, } \\
\text { Eduloc y código QR }\end{array}$ & $\begin{array}{l}\text { Alumnos de } \\
\text { Secundaria }\end{array}$ & $\begin{array}{l}\text { Presentan diferentes } \\
\text { herramientas tecnológicas, con } \\
\text { descripción de las mismas y de } \\
\text { las limitaciones. }\end{array}$ \\
\hline $\begin{array}{l}\text { Casado et al. } \\
\qquad(2016)\end{array}$ & Geocathing & $\begin{array}{l}\text { Alumnos de } \\
\text { Secundaria }\end{array}$ & $\begin{array}{c}\text { Uso del Geocaching en } \\
\text { Educación Física mediante ideas } \\
\text { prácticas. }\end{array}$ \\
\hline $\begin{array}{l}\text { Fernández et al. } \\
\qquad(2015)\end{array}$ & Códigos QR & $\begin{array}{l}\text { Alumnos tercer } \\
\text { ciclo de Primaria }\end{array}$ & $\begin{array}{l}\text { Carrera de orientación urbana } \\
\text { con puntos de interés de la } \\
\text { ciudad y proponiendo retos. }\end{array}$ \\
\hline $\begin{array}{l}\text { Respecto a } \\
\text { excluyendo aque } \\
\text { dos criterios, sob } \\
\text { dentro de la act } \\
\text { contenidos teóric } \\
\text { carácter teórico d }\end{array}$ & $\begin{array}{l}\text { a revisión de experie } \\
\text { as no tan actuales y n } \\
\text { todo: el uso del espa } \\
\text { jidad. Concluyendo la } \\
\text { s y el uso de herram } \\
\text { rante la realización de }\end{array}$ & $\begin{array}{l}\text { cias (véase tabla } \\
\text { educativas. Por lc } \\
\text { io en A.F.M.N y el } \\
\text { relación positiva } \\
\text { entas digitales, ap } \\
\text { a práctica a través }\end{array}$ & $\begin{array}{l}\text { 5) se ha llevado una búsqueda } \\
\text { que se han incluido en relación a } \\
\text { atamiento de contenidos teóricos } \\
\text { xistente entre la adquisición de } \\
\text { tando ideas sobre preguntas de } \\
\text { e códigos QR en las balizas. }\end{array}$ \\
\hline \multicolumn{4}{|c|}{ Tabla 5. Revisión de experiencias educativas } \\
\hline Autoría & Herramienta TIC & $\begin{array}{c}\text { Alumnado } \\
\text { participante }\end{array}$ & Descripción \\
\hline Tejedor (2018) & $\begin{array}{l}\text { E Geocaching y } \\
\text { código QR }\end{array}$ & $\begin{array}{l}4^{\circ} \mathrm{ESO} \text { y } 1^{\circ} \mathrm{de} \\
\text { Bachillerato }\end{array}$ & $\begin{array}{l}\text { Diseño de itinerarios pedestres y } \\
\text { ciclo turistas en el medio natural }\end{array}$ \\
\hline $\begin{array}{l}\text { Ayala-Jiménez } \\
\text { et al. (2017) }\end{array}$ & Código QR & $\begin{array}{l}\text { Estudiantes del } \\
\text { Grado Superior } \\
\text { (TAFAD) }\end{array}$ & $\begin{array}{l}\text { Carrera de orientación para } \\
\text { evaluar los contenidos teóricos } \\
\text { relacionados con este deporte }\end{array}$ \\
\hline $\begin{array}{l}\text { Escaravajal- } \\
\text { Rodríguez } \\
\quad(2017)\end{array}$ & Códigos QR & $\begin{array}{l}\text { Alumnos de } 4^{\circ} \text { de } \\
\text { ESO }\end{array}$ & $\begin{array}{l}\text { Carrera de orientación urbana y } \\
\text { cultural, visitando puntos de } \\
\text { interés de la ciudad. }\end{array}$ \\
\hline $\begin{array}{l}\text { Ternier et al., } \\
\quad(2012)\end{array}$ & Realidad Aumentada & $\begin{array}{l}\text { Estudiantes } \\
\text { Universidad }\end{array}$ & $\begin{array}{c}\text { Juegos con innovaciones } \\
\text { tecnológicas de Realidad } \\
\text { Aumentada más allá del aula } \\
\end{array}$ \\
\hline
\end{tabular}

En lo que se refiere a las investigaciones analizadas (véase tabla 6) se ha tratado de que fueran lo más actuales posible, haciendo hincapié en el análisis de los resultados en referencia al proceso de E-A. Se concluye de manera notable que en todas las investigaciones los elementos digitales al alcance del alumno hacen de elementos potenciadores del aprendizaje con experiencias positivas y enriquecedoras en todos los casos. 
Tabla 6. Revisión de investigaciones educativas

\begin{tabular}{|c|c|c|c|}
\hline Autoría & $\begin{array}{l}\text { Herramientas } \\
\text { TIC }\end{array}$ & $\begin{array}{l}\text { Alumnado } \\
\text { participante }\end{array}$ & Descripción \\
\hline $\begin{array}{c}\text { Escaravajal- } \\
\text { Rodríguez (2018) }\end{array}$ & Códigos QR & $\begin{array}{l}\text { Estudiantes del } \\
\text { Grado Superior } \\
\text { (TAFAD) }\end{array}$ & $\begin{array}{c}\text { Resultados positivos, siendo una } \\
\text { experiencia enriquecedora y } \\
\text { positiva para los alumnos. }\end{array}$ \\
\hline $\begin{array}{l}\text { Gallego et al. } \\
\qquad(2017)\end{array}$ & $\begin{array}{l}\text { Looking4Cache, } \\
\text { C:geo, G. Earth, } \\
\text { QR y R.A }\end{array}$ & $\begin{array}{l}\text { Cuarto curso Grado } \\
\text { de Educación } \\
\text { Primaria en E.F. }\end{array}$ & $\begin{array}{l}\text { Aprendizaje ubicuo a través del } \\
\text { uso de TIC apoya el aprendizaje } \\
\text { de la orientación. }\end{array}$ \\
\hline Franti et al. (2017) & I O-Mopsi & No se especifica & $\begin{array}{c}\text { Estudio de caso y desarrollo } \\
\text { sobre el juego O-Mopsi. Juego de } \\
\text { orientación al aire libre. }\end{array}$ \\
\hline Castro et al. (2016) & Códigos QR & $\begin{array}{l}\text { Alumnos de segundo } \\
\text { ciclo de ESO }\end{array}$ & $\begin{array}{c}\text { Resultados positivos, siendo una } \\
\text { experiencia enriquecedora para } \\
\text { los alumnos. }\end{array}$ \\
\hline Cubillo et al. (2014) & $\begin{array}{l}\text { Realidad } \\
\text { Aumentada }\end{array}$ & $\begin{array}{l}\text { Alumnos de diversos } \\
\text { niveles educativos }\end{array}$ & $\begin{array}{l}\text { La R. A. como un elemento } \\
\text { potenciador de aprendizajes } \\
\text { reales dentro del aula. }\end{array}$ \\
\hline
\end{tabular}

\section{OBJETIVOS}

Los objetivos de este diseño de innovación se relacionan con el problema o situación a mejorar de la realidad educativa, siendo los siguientes:

Objetivos generales

1. Diseñar una aplicación de Orientación Deportiva enfocada a aumentar la adquisición de contenidos y mejorar la experiencia de aprendizaje del alumnado.

2. Evaluar una aplicación de Orientación Deportiva respecto a su usabilidad atendiendo a la adquisición de contenidos, utilidad y motivación para el alumnado de Secundaria.

Objetivos específicos

1.1 Analizar aplicaciones y experiencias que sustenten los criterios de diseño de la aplicación.

1.2 Diseñar un prototipo de la aplicación que mejore el rendimiento académico de los alumnos en Orientación Deportiva.

2.1. Valorar el prototipo en función de los resultados de aprendizaje.

2.2. Analizar el grado de satisfacción de los estudiantes de Secundaria.

\section{MÉTODO}

Las propuestas de aplicación didáctica donde se integra las TIC en Orientación Deportiva (recorridos de orientación) van siguiendo una línea de innovación. El uso de las TIC en E.F. se convierte en una herramienta con un gran potencial y con múltiples posibilidades pedagógicas. Según Gutiérrez \& Monsalve (2016) una aplicación móvil es una herramienta que posee unas dimensiones multifuncionales y se transforma en un soporte para comunicar, impactar, relacionarse, intercambiar... por lo que sería una herramienta de comunicación, gestión y de servicio-producto orientada a proporcionar al usuario las necesidades que demande de forma automática e interactiva.

\subsection{Contexto y participantes}

Esta experiencia apoyada en TIC en orientación deportiva se ha llevado a cabo durante el curso escolar 2019-2020, destacando que no ha sufrido cambios significativos debido a la situación de pandemia producida por el COVID-19, exceptuando el número total de la muestra 
(siendo más escaso) y la utilización solamente del cuestionario como instrumento de evaluación, debido al estado de alarma producido durante el mismo. Por lo que esta propuesta de innovación se ha basado en un contexto de Enseñanza-Aprendizaje, más concretamente en un centro de Educación Secundaria de la provincia de Almería, siendo partícipes, por un lado los profesores del departamento de Educación Física (un hombre y una mujer con más de diez años de experiencia docente) y de Informática (dos hombres interinos) pero todos con experiencia en el uso de apps en el ámbito docente, y 100 alumnos de Secundaria (37 alumnas y 63 alumnos), con edades comprendidas entre 15 años (42 alumnos) y 16 años (58 alumnos) y con características similares en cuanto a curso y nivel académico (segundo ciclo de Secundaria).

La actividad está vinculada de forma directa a los estándares determinados en el currículum oficial de la asignatura según la Ley Orgánica 8/2013 de Educación (LOMCE) y el R.D. $1105 / 2014$, y contribuye a obtener los resultados de aprendizaje perseguidos.

\subsection{Diseño de la propuesta de innovación}

En la elaboración de esta propuesta de innovación me he basado en el Modelo ADDIE, que es considerado por algunos autores (Williams et al., s.f.; Maribe, 2009) como un modelo de carácter genérico y flexible, dado que las fases constituyen los pasos indispensables en todo proceso de diseño. Las fases de la propuesta de innovación se dividen en 3: inicial, central y final, ya que en la segunda se engloba la parte de diseño, desarrollo e implementación. Son las siguientes:

Fase 1: Inicial (análisis): informe de la situación. Inicialmente se ha observado un problema y/o situación a mejorar en la realidad educativa, en relación con la utilización y desarrollo de medios tradicionales en la unidad didáctica (UD) de orientación deportiva, donde los alumnos no alcanzan los objetivos propuestos de la forma deseada. Así, se procede a la identificación de la solución de formación que es una aplicación basada en los criterios de diseño y valoración del alumnado. En la metodología de la intervención con la aplicación se han tenido en cuenta los estándares de aprendizaje que tienen que lograr los alumnos al finalizar la U.D., realizar carreras de orientación en el medio natural en grupo; conocer los contenidos básicos de orientación: mapa, puntos cardinales, balizas..., respetar los elementos que rodean el entorno natural y realizar las sesiones de Orientación Deportiva con el uso de las TIC.

Fase 2: Procesual (diseño, desarrollo e implementación). Engloba tres aspectos muy importantes del proyecto: diseño (elaboración de una aplicación de orientación deportiva que cumpla las expectativas para mejorar y paliar este problema descrito con anterioridad y planificar el proceso de E-A enfocado al alumnado), desarrollo (elaboración y prueba de la aplicación y recursos necesarios, funcionamiento de la app de orientación deportiva, códigos QR, situación de las balizas, funcionamiento tabletas, entorno seguro, desarrollo de las sesiones de orientación, pequeños manuales o tutoriales de descripción para los alumnos, así como realizar una prueba piloto con un pequeño grupo de alumnos) e implementación (plan de aprendizaje, se llevarán a cabo las sesiones de orientación deportiva por parte de los alumnos de 4. ํ ESO, que van a consistir en la realización de carreras de orientación por todo el espacio que comprende el instituto utilizando la app diseñada para tal efecto.

Así, la descripción del uso de la aplicación diseñada en la carrera de orientación es la siguiente:

1) Inicio de la carrera: cada grupo tiene instalada la app en su tableta. Proceden a la apertura de la app y tras pulsar el botón de escanear se les pide que escaneen el primer código QR que es el de inicio de recorrido, para una vez registrado el usuario comenzar la carrera.

2) Empieza a correr el tiempo (cronómetro), el alumno se tiene que dirigir en orden buscando las balizas (de 1 a 10) observando el mapa donde están situadas todas las balizas, no se puede saltar ninguna baliza, localizando y escaneando cada una y contestando cada pregunta (una pregunta por baliza con dos únicas respuestas sobre contenidos de la U.D). El sistema de puntuación es el siguiente:

- Por cada baliza escaneada: + 1 punto

- Por cada pregunta acertada: +2 puntos

- Por cada pregunta equivocada: -1 punto 
3) Una vez escaneadas todas las balizas en orden y contestado a todas las preguntas con su correspondiente retroalimentación (Correcta o incorrecta), se escaneará el código QR final del recorrido, parando el tiempo en el crono y dando por finalizada la actividad. Cada grupo con un tiempo y con una puntuación en el registro.

Fase 3: Final (evaluación). Se pretende valorar la posibilidad de que, a través de la utilización de esta app, puedan mejorar las experiencias de aprendizaje y enriquecer la percepción del entorno y de aprendizaje con una adquisición de los contenidos más constructiva a través de las TIC con los dispositivos digitales (cuestionarios).

\subsection{Diseño de la aplicación}

En el proceso de creación de la aplicación se ha optado por utilizar el programa App Inventor ya que ofrece las siguientes ventajas de utilización en el ámbito escolar según Posada (2019): facilidad en el diseño y programación, tiempo de producción no muy extenso, escalabilidad en su diseño por pantallas o páginas y aplicabilidad, resultando ideal para poder elaborar un producto digital atractivo para el alumnado. El trabajo del prototipo se divide en diferentes bloques donde se van ejecutando las características de cada elemento dependiendo de sus funciones (véase en la figura 1).

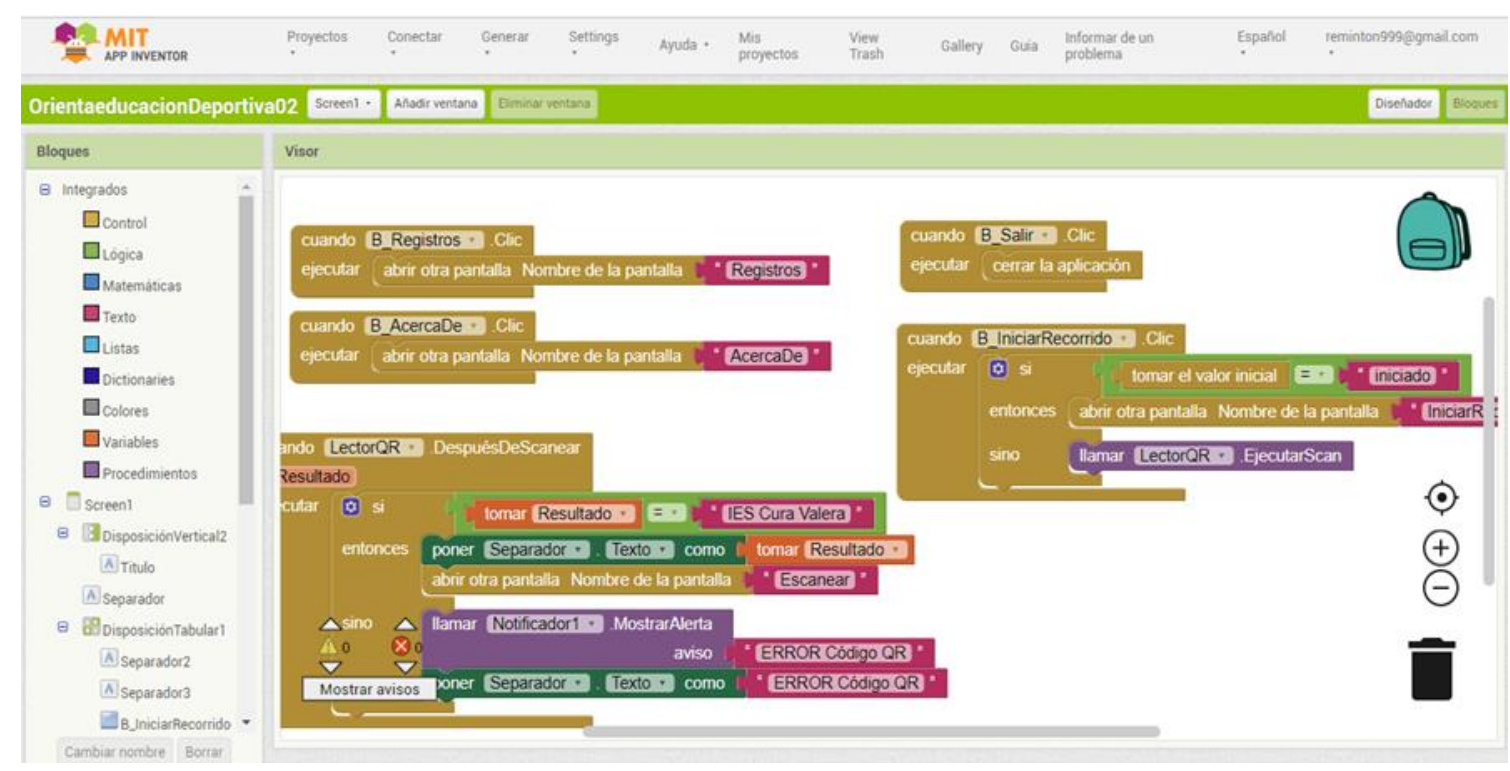

Figura 1. Bloques del prototipo App Inventor

Por tanto, App Inventor es un entorno de desarrollo de software creado por Google para la elaboración de aplicaciones destinadas al sistema operativo de Android que en la actualidad es de los más usados y comercializados. Además, el lenguaje es gratuito y se puede acceder fácilmente desde la web. Las aplicaciones creadas con App Inventor permiten cubrir un gran número de necesidades básicas en un dispositivo móvil, por lo que son muy intuitivas para el alumno como se puede ver en el prototipo final de la pantalla de inicio de la app (véase figura 2). 


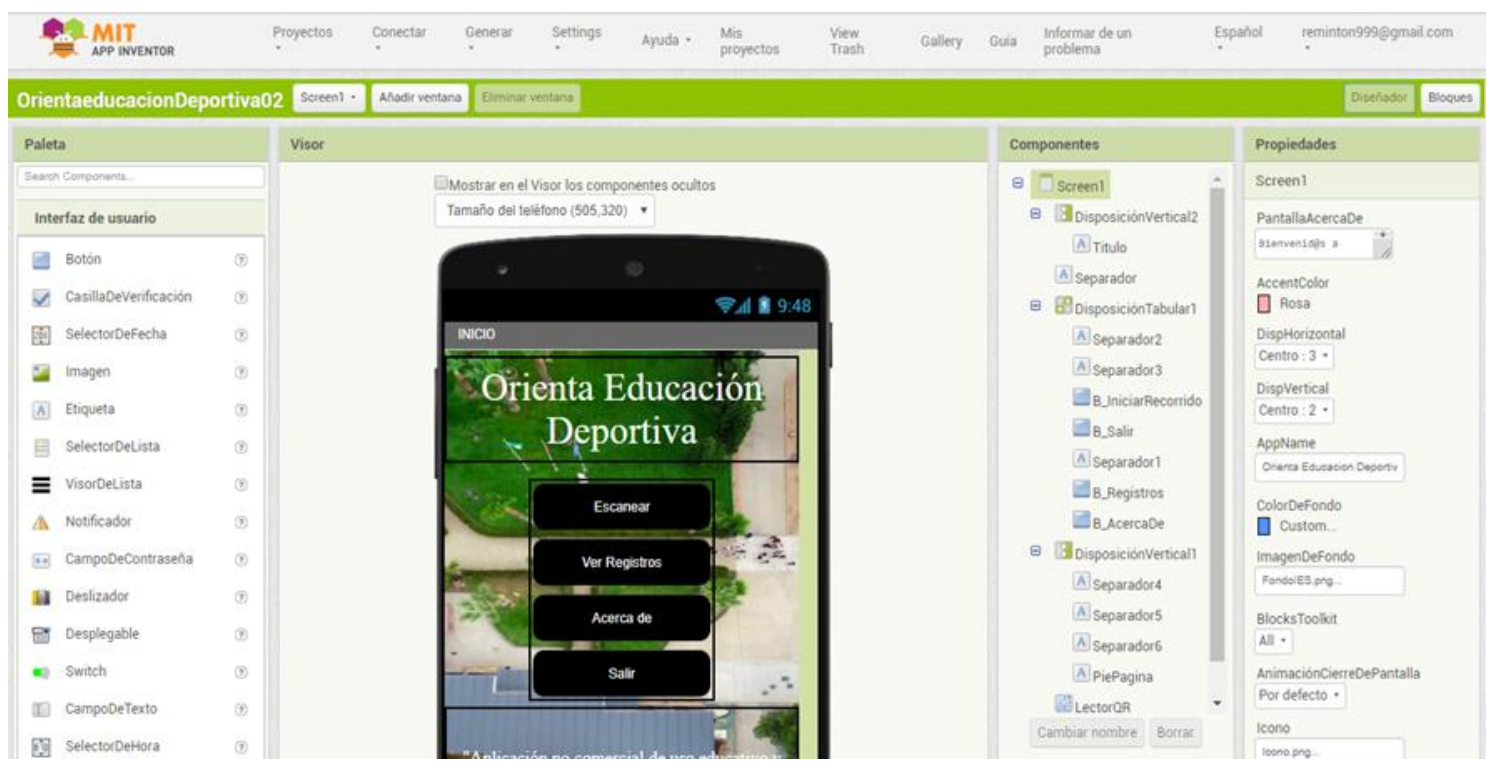

Figura 2. Prototipo final de la pantalla de inicio de la App

\subsection{Instrumento de evaluación}

Al ser un trabajo de innovación, cabe la posibilidad de no abordar el uso de instrumentos de recogida de datos con tanto rigor metodológico como sí debería hacerse en una investigación, pues en situaciones de innovación lo realmente importante y relevante es la obtención de información de carácter práctico para mejorar el contexto concreto de aplicación del prototipo desarrollado (Prendes \& González, 2017). Así, como instrumento de evaluación se utiliza el cuestionario, ya que es económico, incluye las mismas preguntas para todos los participantes (alumnos) y asegura el anonimato (Prendes \& González, 2017).

Al ser adolescentes, a los padres de cada alumno se les hizo entrega de una autorización para que dieran su consentimiento respecto al cuestionario que se les iba a pasar a sus hijos. Así, se ha tratado de forma cuantitativa (primer y segundo bloque) como cualitativa (tercer bloque) donde por un lado, se ha utilizado el cuestionario de usabilidad de Hernane Borges (2002) respecto a la aplicación de Orientación Deportiva (características de la aplicación) donde se tiene un coeficiente de fiabilidad alfa de Cronbach de 0,889, por lo que el primer bloque de 21 preguntas corresponde al cuestionario de dicho autor sin modificación en las mismas organizado según las siguientes dimensiones: estructura, operación, información, apariencia, intuición, contenido y experiencia de la aplicación. Posteriormente se añade un segundo bloque de 9 preguntas donde se trata la experiencia del usuario en relación al proceso de E-A del alumno respecto a la U.D. de Orientación Deportiva, para valorar el prototipo en función de los resultados de aprendizaje y analizar el grado de satisfacción de los estudiantes de Secundaria de elaboración propia. Finalmente se ha dejado un apartado final (pregunta abierta), analizado con el Programa Atlas.ti 8, donde los alumnos pueden expresar sus opiniones, sugerencias sobre la propuesta de innovación llevada a cabo.

El modelo de cuestionario con las 30 preguntas realizadas a los alumnos se realizó mediante el envío del enlace por correo electrónico. Se aplicó con un formulario de Google Drive ya que es una herramienta gratuita, sencilla de utilizar y usar, almacena los datos de forma segura posibilitando el poder trabajar con otras personas desde distintos lugares, para crear documentos online y compartirlos, siendo una de las herramientas para trabajar en grupo más valoradas por los alumnos (Delgado \& Casado, 2012).

En el cuestionario nos encontramos con descriptores de evaluación en cada pregunta. Una serie de criterios de evaluación-medición de logro respecto a la usabilidad de la app y al proceso de E-A del alumnado en referencia al uso de la misma (Escala Likert). A la hora de rellenar el cuestionario se le pide al alumno que marque en cada pregunta la casilla que mejor represente su opinión (grado de acuerdo) de la siguiente manera:

- 1: estar totalmente en desacuerdo 
- 2: en desacuerdo

- 3: ni de acuerdo ni en desacuerdo

- 4: de acuerdo

- 5: totalmente de acuerdo

\section{RESULTADOS}

El análisis de datos se muestra a través de porcentajes, lo cuales, sintetizan de manera clara y concisa las respuestas de los participantes. Los resultados los estructuramos en base a las 30 preguntas del cuestionario más las opiniones y sugerencias de los alumnos.

\subsection{Bloque 1: Usabilidad de la App de Orientación Deportiva (21 preguntas)}

En líneas generales se tienen valores muy positivos tanto en la información al usuario (sistema de ayuda, retroalimentación y búsqueda de información), así como en apariencia y navegación con valores que superan el $80 \%$ de satisfacción. Respecto a la estructura de la aplicación hay 3 dimensiones a evaluar (véase figura 3). Los alumnos valoran la organización estructural de los elementos con una visión positivista con más del $89 \%$ de grado satisfactorio, siendo sólo $2 \%$ totalmente en desacuerdo y $9 \%$ con valores neutros. Respecto a la densidad de los elementos en la estructura en cambio, sobre el $47 \%$ del alumnado está con un grado de disconformidad ( $6 \%$ totalmente en desacuerdo y $41 \%$ en desacuerdo) en lo que se refiere a la excesiva densidad de elementos en la aplicación, mientras que $33 \%$ tienen valores neutros, un $20 \%$ considera que hay excesivos elementos. Por último, la constancia en la distribución de elementos se identifica satisfactoriamente con valores de $60 \%$ y $26 \%$ positivos, con sólo $2 \%$ en desacuerdo y $12 \%$ neutros.

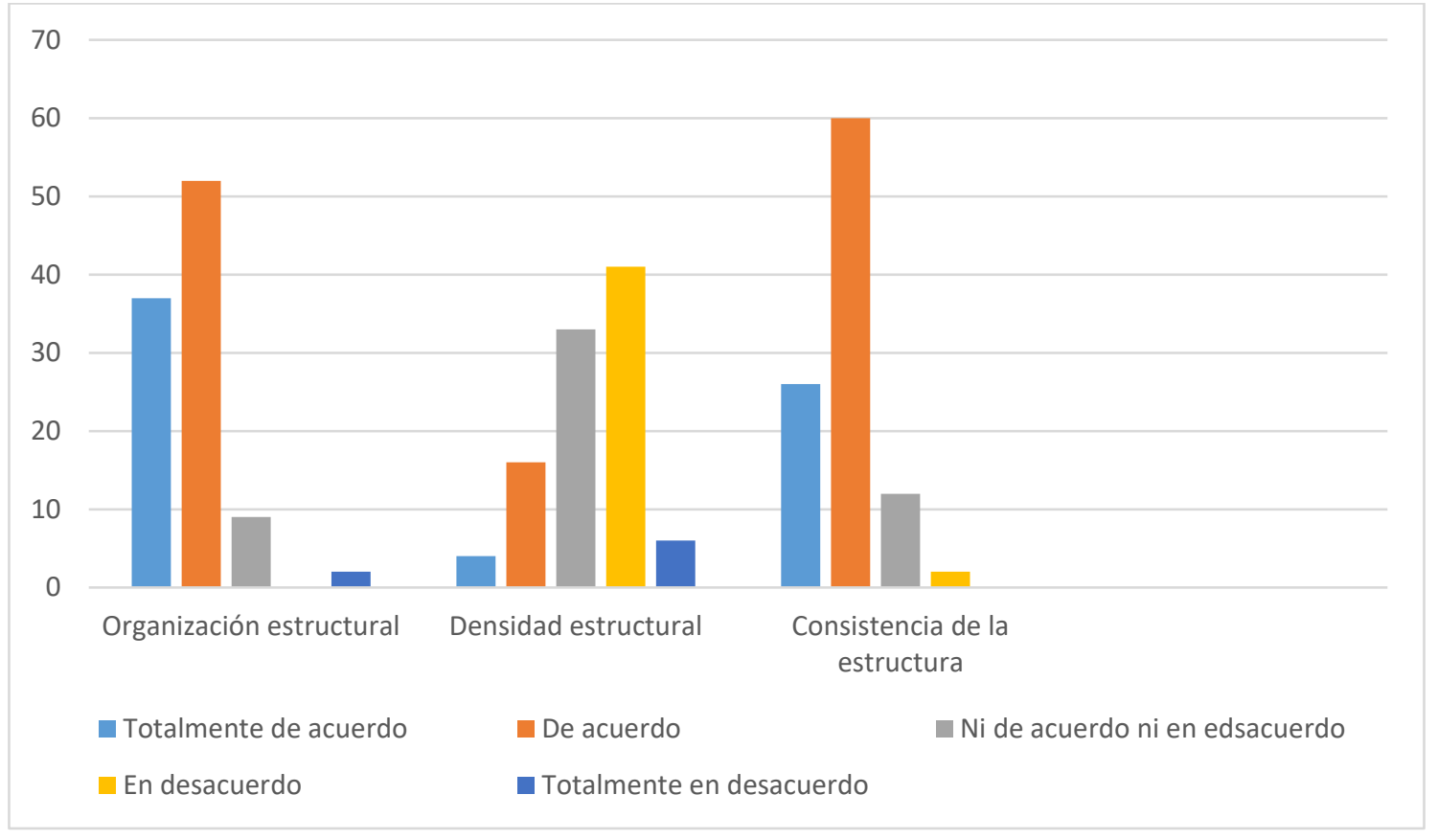

Figura 3. Opinión sobre la estructura

En lo que respecta a la operación de la aplicación hay 7 dimensiones a evaluar (véase figura 4): la navegabilidad (los alumnos consideran que el recorrido por el contenido de la aplicación es fácil con un $85 \%$ conforme, y solamente muestran su disconformidad en un $2 \%$, quedando 13 $\%$ en valores neutros), interactividad (con valores positivos de un $89 \%$ mientras que no es considerada buena sólo un $3 \%$ y un $8 \%$ que no está de acuerdo ni en desacuerdo), accesibilidad (las acciones que solicita la aplicación son fáciles de ejecutar según la mayoría del alumnado con un $89 \%$, mientras que sólo un $1 \%$ estaría en desacuerdo y un $10 \%$ en valores neutros), sistema de indicación (destaca un $78 \%$ de conformidad, pero un $21 \%$ se muestra neutro ante este ítem y $1 \%$ en desacuerdo), desempeño del sistema (la velocidad de funcionamiento es 
considerada muy positiva en un $88 \%$ mientras que sólo un $4 \%$ muestran su disconformidad y un $8 \%$ no estaría ni de acuerdo ni en desacuerdo), fiabilidad ( muestra valores más negativos ante este ítem con un $64 \%$ de disconformidad con $18 \%$ totalmente en desacuerdo, $19 \%$ con valores neutros y $17 \%$ de forma satisfactoria) y consistencia de la operación (los alumnos consideran que sigue un estándar a lo largo de la aplicación con valores positivos en el $82 \%, 14$ $\%$ valores neutros y solamente un $6 \%$ de disconformidad).

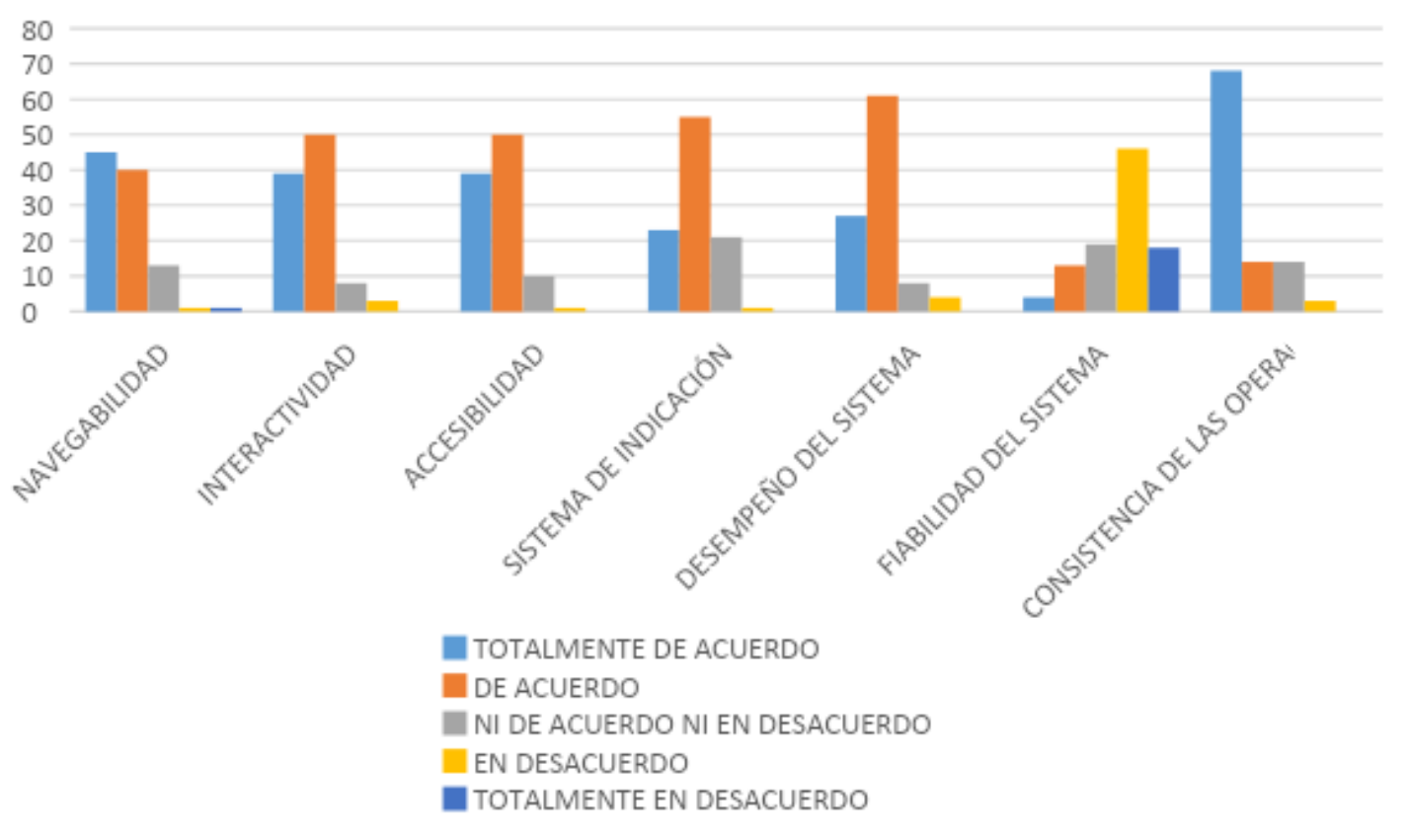

Figura 4. Opinión sobre la operación

Respecto al contenido hay cuatro dimensiones que se han tenido en cuenta (véase figura 5). La distribución y organización del contenido en cuanto a textos, imágenes tiene una valoración buena ya que $91 \%$ muestra grado satisfactorio, sólo el $2 \%$ insatisfactorio, siendo el $7 \%$ valores neutros. La densidad de contenidos en cambio, mantiene valores neutros con un $33 \%$, mientras que en valores de conformidad en cuanto a que la información es demasiado extensa hay un 24 $\%$ y solamente sobre un $43 \%$ considera adecuada la cantidad de contenidos expuesta en la app. Los alumnos consideran la fiabilidad del contenido de forma positiva con un $76 \%$ respecto a que no hay errores, mientras que el $20 \%$ se muestra neutro y el 4 no conforme. Por último, la información que se presenta en la app es considera fácil de entender por el $84 \%$ del alumnado, con sólo un $3 \%$ de disconformidad y $13 \%$ ni de acuerdo ni en desacuerdo. 


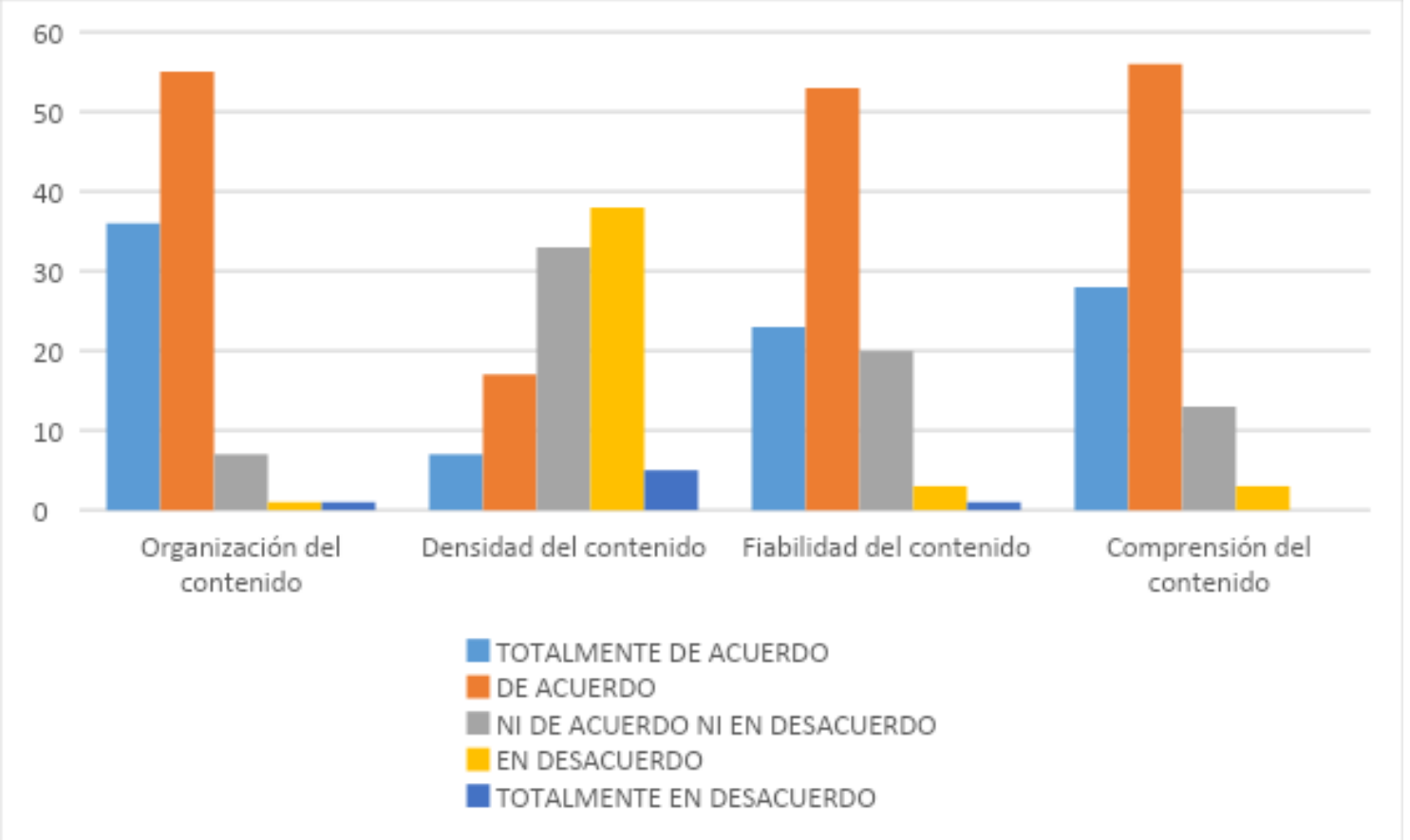

Figura 5. Opinión sobre el contenido

Respecto a la experiencia del usuario con la app se valora de forma muy positiva sin datos de disconformidad, destacando que la aplicación en líneas generales como se puede observar en la figura 6 , le ha parecido buena a un $92 \%$ del alumnado, siendo sólo el $1 \%$ el que se muestra en desacuerdo y un $7 \%$ neutro.

100 respuestas
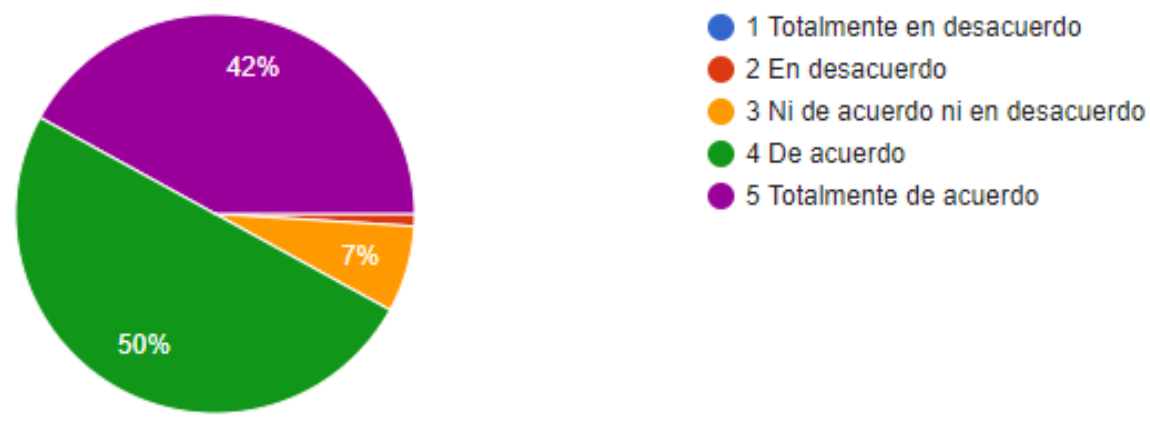

Figura 6. Opinión general de la aplicación

\subsection{Bloque 2: experiencia del alumnado respecto al proceso de E-A en las carreras de Orientación Deportiva (9 preguntas)}

En este bloque se tienen valoraciones muy positivas respecto a las dimensiones relacionadas con elementos del medio ambiente con más de un $90 \%$. Respecto a los elementos clave en las carreras de orientación hay 3 dimensiones a evaluar (véase figura 7). Los encuestados creen que la aplicación ayuda a conocer los elementos clave de orientación deportiva en un $87 \%$, siendo sólo $2 \%$ de disconformidad y $11 \%$ valores neutros. Además, valoran positivamente que el mapa de la aplicación permita diferenciar la simbología que en él se presentan en un $85 \%$, siendo el $11 \%$ neutral, $3 \%$ en desacuerdo y $1 \%$ totalmente en desacuerdo. Los alumnos consideran en un $95 \%$ que la realización de carreras de orientación les facilita la adquisición de contenidos, mientras que sólo el $1 \%$ está de desacuerdo y el $4 \%$ neutral. 


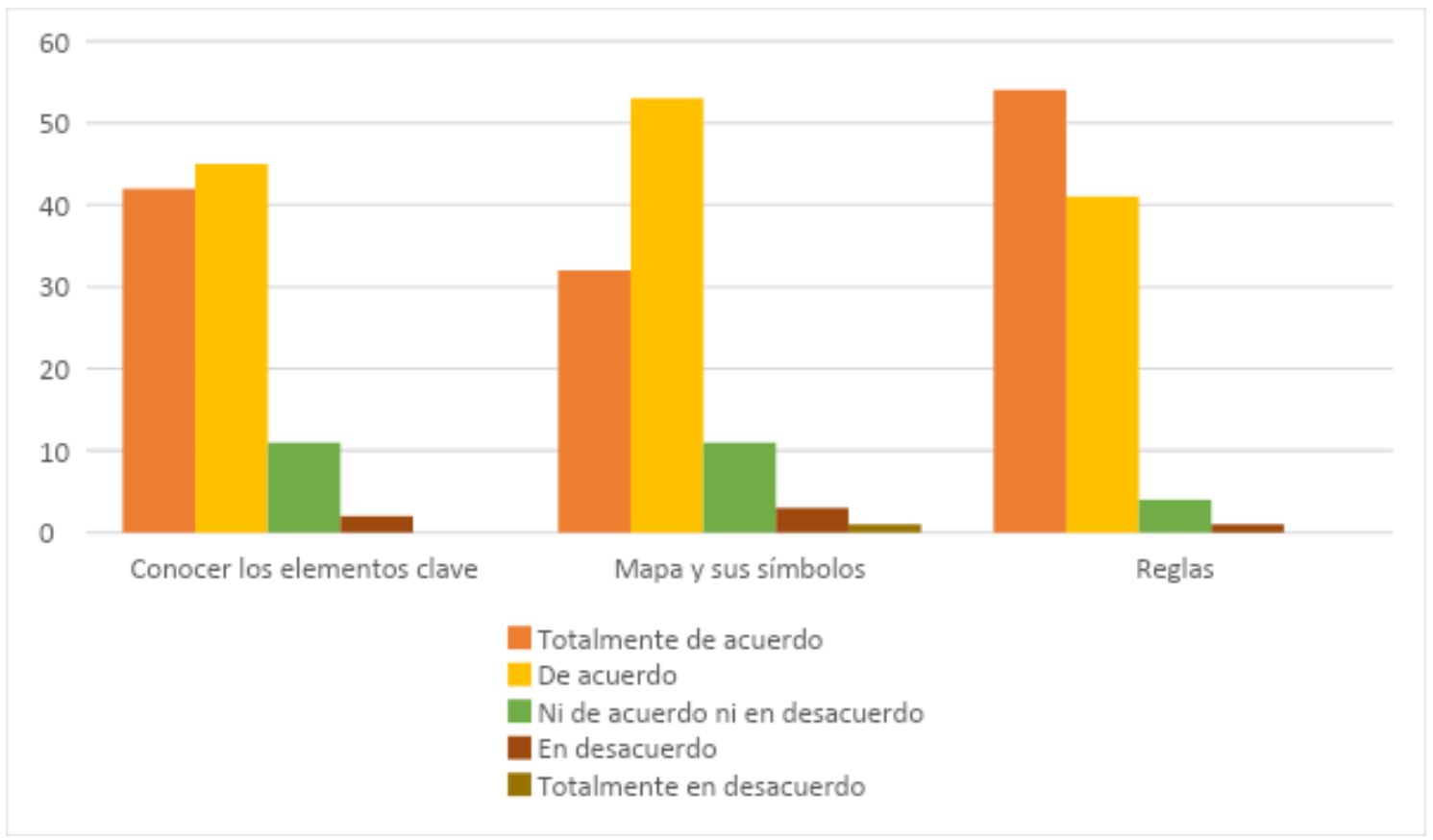

Figura 7. Opinión sobre los elementos clave en las carreras de orientación deportiva

Los encuestados consideran que las TIC les han ayudado en el desarrollo de las carreras de orientación, mostrando un grado de acuerdo general de un $85 \%$, siendo nulo el grado de disconformidad y $15 \%$ de neutralidad como se observa en la figura 8 .

100 respuestas

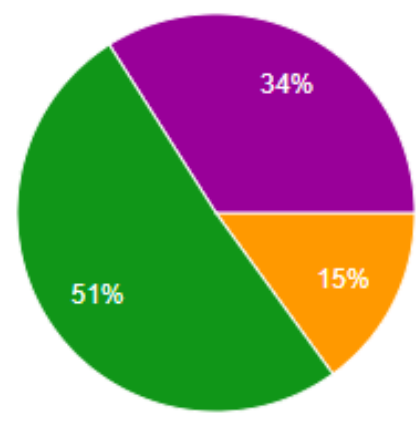

1 Totalmente en desacuerdo

2 En desacuerdo

3 Ni de acuerdo ni en desacuerdo

4 De acuerdo

5 Totalmente de acuerdo

Figura 8. Opinión sobre la relación de las TIC y carreras de orientación

Los alumnos consideran que la aplicación les ha ayudado a adquirir conocimientos nuevos con más del $85 \%$ de conformidad, y como se puede ver en la figura 9 en líneas generales el alumnado tiene una visión positiva de la U.D. de Orientación Deportiva en su globalidad de conjunto con un $92 \%$ de valores positivos (65\% totalmente de acuerdo), solo $1 \%$ de disconformidad y $7 \%$ que no están ni de acuerdo ni en desacuerdo. 


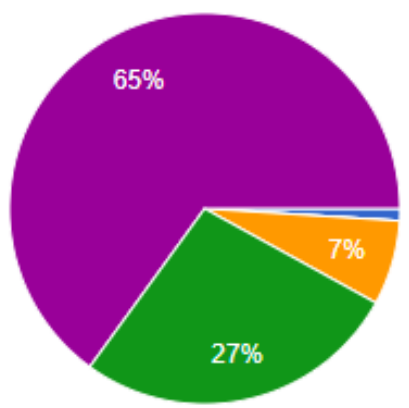

1 Totalmente en desacuerdo

2 En desacuerdo

3 Ni de acuerdo ni en desacuerdo

4 De acuerdo

5 Totalmente de acuerdo

Figura 9. Opinión general de la U.D. de orientación deportiva

\subsection{Opiniones y sugerencias realizadas por el alumnado}

Para analizar la información de forma cualitativa, se ha utilizado el programa Atlas.ti8. Se ha observado que todos los comentarios de los alumnos son de carácter positivo hacia la usabilidad de la aplicación, efectuando el $100 \%$ de apreciaciones con grado de acuerdo en lo que respecta a la mayor adquisición de contenidos respecto al deporte en el medio natural de Orientación Deportiva a través del uso de la app, dándole importancia a factores como: el trabajo en equipo, valores de cooperación, compañerismo, respeto por el medio natural..., resaltando la buena experiencia, lo divertido que ha sido y el aprendizaje de nuevos contenidos.

\section{DISCUSIÓN}

Esta propuesta de innovación trata de dar respuesta a los objetivos planteados, por lo que se establece lo siguiente:

Los continuos avances tecnológicos repercuten directamente en el proceso educativo y, por consiguiente, en las estrategias y métodos que utilizan los docentes para pretender que sus alumnos adquieran un aprendizaje significativo. Según Colás et al. (2018), se lleva realizando un esfuerzo muy grande a nivel europeo, nacional y autonómico para el desarrollo e impulso del uso de las TIC. La educación física y su profesorado están llamados a seguir procesos de inmersión digital, asociados al uso de tecnología, especialmente la móvil (Díaz, 2019).

Por tanto, la realización de actividades de orientación en el medio natural con apoyo de una herramienta digital (aplicación móvil) como posibilidad educativa en el ámbito de la educación formal, constituye una importante cualidad a esta disciplina en la consolidación de los procesos educativos de nuestros alumnos. Según Escaravajal (2018) las tecnologías están muy presentes en orientación deportiva, existiendo una gran variedad de Apps que pueden facilitar las posibilidades para su desarrollo y disfrute en el medio natural.

Así, el interés y motivación del alumnado hacia la práctica de Orientación Deportiva ha sido más que evidente desde mucho antes de la práctica debido al uso de dispositivos móviles incluidos en ella, gracias a su carácter digital, componente de novedad y curiosidad y contenido realizado en el medio natural. El dinamismo de la tecnología móvil y el uso generalizado de dispositivos, especialmente teléfonos inteligentes que permiten adoptar nuevos modelos pedagógicos más motivantes para el alumnado (Traxler, 2016).

Los motivos por los que la actividad con TIC presenta un atractivo formativo añadido a la orientación deportiva en el medio natural según Rodríguez et al. (2017) se ha visto reflejada en nuestra propuesta de innovación con comportamientos del alumnado cooperativos y de trabajo en equipo ante situaciones poco habituales para ellos, para lograr un objetivo común con posibilidad de éxito, con una motivación añadida por el uso de las TIC. La innovación educativa en el proceso de E-A pasa por la utilización de herramientas digitales en el aula (Tejedor, 2018). 


\section{CONCLUSIONES}

Tras el análisis de los resultados se puede observar, por un lado, que los alumnos valoran la aplicación de forma muy positiva en referencia a las características de la misma con valores de conformidad por encima del $85 \%$ en organización estructural y del contenido, navegabilidad, accesibilidad y búsqueda de información entre otros aspectos; teniendo una experiencia en general interesante y favorable en un $89 \%$ pareciéndole una aplicación buena al $92 \%$. En cambio, valoran de forma neutra entre el $45 \%-65 \%$ de conformidad aspectos como densidad estructural y de contenido y fiabilidad del sistema. Por otro lado, la experiencia del alumnado respecto al proceso de E-A en las carreras de Orientación Deportiva tiene aspectos significativos con valores muy altos entre el $87 \%$ y $93 \%$ de conformidad tanto en la adquisición de contenidos y valores sobre los elementos de las carreras de Orientación Deportiva y valores de deportividad, salud y respeto del medio natural, así como en el desarrollo de la competencia digital con un 87 $\%$. Es de señalar que el $92 \%$ ve la U.D de Orientación Deportiva como divertida e interesante en líneas generales destacando el trabajo en equipo como factor primordial a la hora de llevar a cabo la actividad.

Con la implantación de esta propuesta de innovación se intenta fomentar el uso de las TIC para el desarrollo de la competencia digital en el centro educativo haciendo que convivan y se favorezcan el uno del otro. La idea es emplear las TIC como recurso generador de gran cantidad de funciones en el ámbito educativo para mejorar el proceso de E-A. Una de las mayores ventajas de usar TIC consiste en la posibilidad de romper las barreras espacio-temporales que tanto condicionan la enseñanza y el aprendizaje de la Educación Física (Barahona, 2012). Se considera que los recursos tecnológicos pueden ser una excelente herramienta de apoyo a la hora de trabajar la orientación en el ámbito educativo (Escaravajal, 2018). También se pone en evidencia cómo el propio profesorado se favorece también de estas herramientas en dicho proceso. El uso de las TIC en los procesos didácticos permite innovar en los contenidos y en la forma de enseñar la materia, aportando numerosos beneficios a profesores y a estudiantes (Díaz, 2018).

\subsection{Limitaciones y futuro}

Tras el desarrollo e implantación de la propuesta se ha llegado a la conclusión de que todavía quedan aspectos en líneas generales de la usabilidad de la aplicación que tienen que aumentar su grado de calidad respecto a los criterios de diseño de la aplicación.

Si nos centramos en la estructura de la aplicación, se deben considerar aspectos modificables de distribución de los elementos tanto en la pantalla principal como en las pantallas secundarias, siendo la densidad de los mismos un aspecto a mejorar. Además, en consonancia a su fiabilidad y cantidad de contenidos e información expuesta a lo largo de las pantallas (con valores por debajo del $60 \%$ de conformidad del alumnado) se intentará dar más claridad, sencillez e intuición en su manejo, tanto en el formato de texto, color de la fuente y forma de los botones.

Así, las fortalezas de la aplicación respecto a las características de usabilidad son: la organización estructural y de contenidos en sus pantallas (elementos muy sencillos y claros como los botones, texto, imágenes...), la facilidad en la navegabilidad (pantalla principal muy intuitiva con poca información, pero muy corta y clara), accesibilidad (botones de acceso muy claros derivando a las funciones principales de la aplicación) y búsqueda de información fácil de encontrar dependiendo de los objetivos de la actividad;

Respecto al proceso de E-A la relación práctico-teórica de contenidos de la U.D. de Orientación Deportiva fue muy satisfactoria, ya que con la introducción de preguntas en cada baliza se intentaba completar el desarrollo de los contenidos dados en clase sobre esta temática. Se pudo valorar como se puede mejorar tanto en la cantidad y dificultad de las preguntas, así como en la modificación de algunas que creaban cierta confusión o ambigüedad.

Durante el desarrollo de las sesiones de orientación se detectaron varios problemas de funcionamiento, observando que en los grupos más numerosos el manejo de la aplicación se llevaba principalmente a cabo por uno o dos miembros del grupo, dejando a otros alumnos sin interaccionar con la herramienta digital. Para futuras utilizaciones de la aplicación se fomentará 
el trabajo en grupo para el desarrollo de valores de cooperación, compañerismo, deportividad... pero con grupos reducidos formados por 2-3 alumnos.

En definitiva, pretendemos mejorar la aplicación de diferentes maneras. Inicialmente centrándonos en las debilidades detectadas en esta propuesta de innovación, es decir, densidad y distribución de elementos, considerando mejorar la distribución y desarrollo de elementos en la pantalla principal, así como en el mapa, balizas y preguntas realizadas. Posteriormente centrándonos en los pilares básicos de una aplicación para el ámbito educativo según Posada (2019) haciendo más partícipe al alumno en el diseño de la aplicación, dejando que se involucre más participando desde dentro en su desarrollo. Finalmente se piensa que realizando más pruebas pilotos con nuestros alumnos se mejorará de forma notable en futuras carreras de orientación.

\section{ENLACES}

A la carpeta de diseño de la aplicación: https://bit.ly/3fNOcRP

Al instrumento de evaluación (preguntas): https://bit.ly/3dFcrNK

Al instrumento de evaluación (respuestas): https://bit.ly/3dGQWff

\section{REFERENCIAS BIBLIOGRÁFICAS}

Abril, C.A. \& Acosta E.L. (2015). Aplicación de las TIC como herramienta didáctica para la enseñanza - aprendizaje de la técnica básica del balonmano con los niños del grado quinto del colegio Nydia Quintero de Turbay I.E.D [Tesis de pregrado, Universidad Libre]. Repositorio Institucional Unilibre. https://bit.ly/2CHbgyR

Alcaraz, V., Sánchez, A. J., \& Grimaldi, M. (2018). El gran juego salvando a gea: gamificación y nuevas tecnologías en actividades físicas en medio natural. EmásF, Revista Digital de Educación Física, 9(54), 148-158. https://bit.ly/3iMFRew

Aubusson, P., Schuck, S., \& Burden, K. (2016) Mobile learning for teacher professional learning: benefits, obstacles and issues, $A L T-J, 17(3), 233-247$. https://bit.ly/3gkdbsn

Ayala, J. D., Escaravajal, J. C., Otálora, F. J., Ruiz, Z. M. \& Nicolás, J. (2017). Carrera de orientación con códigos QR en Educación Física. Espiral. Cuadernos del Profesorado, 10(21), 132-139. https://dx.doi. org/10.25115/ecp.v10i21

Aznar, I., Cáceres, M.P., Trujillo, J.M., \& Romero, J.M. (2019). Impacto de las apps móviles en la actividad física: un meta-análisis. Retos: nuevas tendencias en educación física, deporte y recreación, 36, 52-57. https://bit.ly/3axrzCp

Barahona, J. (2012). La enseñanza de la Educación Física implementada con TIC. Revista Educación Física y Deporte, 31(2), 1047-1056. https://doi.org/10.28997/ruefd.v0i11.1

Casado, O. M., Hernando, A., Hortigüela, D., \& Pérez, A. (2017). IOrienteering. Tándem: Didáctica de la Educación Física, 55, 75-76. https://bit.ly/3edouzZ

Casado, O. M., Pérez, A. \& Hortigüela, D. (2016). Geocaching. Tándem: Didáctica de la Educación Física, 53, 77-78. https://bit.ly/3iLcEAB

Castro, N. \& Gómez, I. (2016). Incorporación de los códigos QR en la Educación Física en Secundaria. Retos. Nuevas tendencias en Educación Física, Deporte y Recreación, 29, 114119. https://bit.ly/3fclity

Colás, M.P., De Pablos, P. \& Ballesta, J. (2018). Incidencias de las TIC en la enseñanza en el sistema educativo español: una revisión de la investigación. Revista de Educación a Distancia, 56(2), 1-23. https://bit.ly/3a83hsW

Cubillo, J., Martín, S., Castro, M. \& Colmenar, A. (2014). Recursos digitales autónomos mediante realidad aumenta. Ried, 17(2), 241-274.https://doi.org/10.5944/ried.17.2.12686 
Díaz, J. (2018). Mejorar el aprendizaje en acción integrando mobile learning en la educación física. Tándem: Didáctica de la educación física, 60, 69-73. https://bit.ly/3gbllOR

Díaz, J. (2019). Retos y oportunidades de la tecnología móvil en la educación física. Retos, 37(37), 763-773. https://bit.ly/2lekt4i

Escaravajal, J. C. (2017). La orientación deportiva como medio interdisciplinar. Trances, 9(6), 885-908. https://bit.ly/3s12ajO

Escaravajal, J. C. (2018). Los Códigos QR en Educación Física: Carrera de Orientación. Pensar en Movimiento: Revista de Ciencias del Ejercicio y la Salud, 16(1), 1-14. https://bit.ly/3dOJYoN

Escaravajal, J.C. \& Baena, A. (2019). Las Aplicaciones Tecnológicas en el deporte de Orientación y en Educación Física. Habilidad Motriz. Revista de Ciencias de la Actividad Física y del Deporte, 53. 28-39. https://doi.org/10.24310/riccafd.2019.v8i1.5770

Espuny, C., Gisbert, M. Coiduras, J. \& González, J. (2012). El coordinador TIC en los centros educativos: funciones para la dinamización e incorporación didáctica de las TIC en las actividades de aprendizaje. Píxel-Bit. Revista de Medios y Educación, 41, 7-18. https://bit.ly/2ZS1q4v

Fernández, R., Herrera-Vidal, J. I., \& Navarro, R. (2015). Las TIC como recurso en la didáctica de la educación física escolar. Propuesta práctica para la educación primaria. EmásF, Revista Digital de Educación Física, 6(35), 58-69. https://bit.ly/2BJMQEr

Franti, P., Mariescu-Istodor, R., \& Sengupta, L. (2017). O-Mopsi: Mobile Orienteering Game for Sightseeing, Exercising, and Education. ACM Trans. Multimedia Comput. Commun. Appl., 13(4), 1-25. https://doi. org/10.1145/3115935

Gallego,V., Muñoz, J. A., Arribas, H. \& Rubia, B. (2017). La orientación en el medio natural: aprendizaje ubicuo mediante el uso de tecnología. Movimiento, 23(2), 755-770. https://doi.org/10.22456/1982-8918.71682

Generelo, M. (2010). Las nuevas tecnologías y su aplicación en educación física. Libro de Actas $\checkmark$ Congreso Internacional XXVI Nacional de Educación Física: Docencia, innovación e investigación física Barcelona, 172-181. https://bit.ly/3eXIND2

Gutiérrez, M.V. \& Monsalve, D.A. (2016). Diseño de una aplicación móvil que actualiza procesos de enseñanza aprendizaje permitiendo la adquisición de conocimientos en capacidades físicas condicionales y deportes. Revista Reflexiones, 1(6), 89-104. https://doi.org/10.18041/2011-5768/reflexiones.0.2016.4352

Hernane Borges, P. (2002). Análisis experimental de los criterios de evaluación de usabilidad de aplicaciones multimedia en entornos de educación y formación a distancia [Tesis doctoral, Universidad Politécnica de Cataluña]. TDX. https://bit.ly/3jFwGwO

Lavay, B., Sakai, J., Ortiz, C., \& Roth, K. (2015). Tablet technology to monitor physical education IEP goals and benchmarks. Journal of Physical Education, Recreation \& Dance, 86(6), 1623. https://bit.ly/2VHB2Ja

Ley Orgánica 8/2013, de 9 de diciembre, para la Mejora de la Calidad Educativa (LOMCE). Boletín Oficial del Estado (BOE). 10 de diciembre de 2013, 1-64. https://bit.ly/3fhgu2m

Maribe, R. (2009). Instructional Designe: The ADDIE Aproach. https://doi.org/10.1007/978-0-387$\underline{09506-6 \quad 3}$

Ortega, J, Padilla, C., Moreno, M., Pérez, J. \& Gomez, M. (2007). Nuevas tecnologías aplicadas a la cuantificación en fútbol. Revista Kronos, 12, 22-28. https://bit.ly/2D6qtt8

Posada, F. (2019). Creando aplicaciones para móviles Android con MIT App Inventor 2. Observatorio de tecnología educativa, 12. https://bit.ly/3wAdret

Prendes Espinosa, M.P. \& González Calatayud, V. (2017). Trabajo Fin de Máster en Tecnología Educativa: orientaciones para la elaboración y criterios de calidad. Servicio de Publicaciones de la Universidad de Murcia. https://bit.ly/31XTC2A 
Real Decreto 1105/2014, de 26 de diciembre, por el que se establece el currículo básico de Educación Secundaria Obligatoria y del Bachillerato. Boletín Oficial del Estado, 3 de enero de 2015, 1-35. https://bit.ly/3gKhdcK

Reyes Velandia, J. C. (2019). Apropiación conceptual de los fundamentos técnicos del voleibol en dos ambientes de aprendizaje: con mediación de las TIC uso del video frente al aprendizaje tradicional [tesis de maestría, Universidad Pedagógica y Tecnológica de Colombia, Duitama]. https://bit.ly/32WdxAG

Sánchez Álcaraz, B. J. (2015). Mejora de la competencia motriz para los deportes de raqueta a través del vídeo digital en las clases de Educación Física. Revista científica electrónica de Educación y Comunicación en la Sociedad del Conocimiento, 15(1), 76-88. https://bit.ly/2O7Ah8a

Santos, P. et al. (2011). QuesTInSitu: From tests to routes for assessment in situ activities. Computers \& Education, 57(4), 2517-2534. https://doi.org/10.1016/i.compedu.2011.06.020

Tejedor, J. C. (2018). Las actividades físicas en el medio natural a través de un proyecto de innovación educativa en el IES Tomás y Valiente. EmásF, Revista Digital de Educación Física, 9(54), 29-40. https://bit.ly/3fpEoll

Ternier, S. et al. (2012). ARLearn: Augmented Reality meets Augmented Virtuality. Journal of Universal Computer Science, 18(15), 21-64. https://doi.org/10.3217/jucs-018-15-2143

Traxler, J. (2016). Inclusion in an age of mobility. Research in Learning Technology, 24, 1-13. https://bit.ly/39QfYZi

Turpo, O. (2018). La usabilidad pedagógica en la formación del profesorado: un estudio de caso. Revista Espacios, 39(15). https://bit.ly/3/JiP8B

Vázquez-Cano, E., \& Sevillano, M L. (2015). Dispositivos digitales móviles en Educación. Revista de Currículum y Formación de Profesorado, 20(1), 20-21. https://bit.ly/2lfGwaX

Williams, P., Lynne, S., Sangrà, A., \& Guàrdia, L. (s.f.). Fundamentos del diseño técnico pedagógico en e-learning. Modelos de diseño instruccional. UOC Editorial.

\section{INFORMACIÓN SOBRE LOS AUTORES}

\section{Remigio Pérez Rabadán}

IES Cura Valera de Húercal Overa

Profesor de Educación Secundaria de Educación Física desde el 2008, actualmente en el IES Cura Valera de Húercal-Overa (Almería) ejerciendo como profesor de Educación Física Bilingüe, Jefe de Departamento de Educación Física y Coordinador del Programa Forma Joven y Hábitos de Vida Saludables. Socio de Centro Deportivo Playfitness en la localidad de Albacete (CastillaLa Mancha) desde el 2013. Estudiante del Máster Universitario en Tecnología Educativa: eLearning y Gestión del Conocimiento durante el curso escolar 2019-2020. Certificado de la Escuela Oficial de Idiomas Nivel C1 Inglés en el año 2019. Doctorado en Educación: Nuevas Perspectivas. Inicio de estudios en 2008 Licenciado en Ciencias de la Actividad Física y del Deporte en el Campus Universitario de Toledo (Castilla- La Mancha) de 2005 a 2007. Diplomado en Educación Física en Campus Universitario de Albacete (Castilla- La Mancha) de 2002 a 2005. Líneas de investigación relacionadas con el diseño de herramientas digitales didácticas en torno al ámbito educativo (Tecnología Educativa). Enlace web propio: https://www.remiprofesoref.es/

Los textos publicados en esta revista están sujetos a una licencia de Reconocimiento 4.0 España de Creative Commons. Puede copiarlos, distribuirlos, comunicarlos públicamente y hacer obras derivadas siempre que reconozca los créditos de las obras (autoría, nombre de la revista, institución editora) de la manera especificada por los autores o por la revista. La licencia completa se puede consultar en:Licencia Creative Commons Atribución-NoComercial-Compartir por igual 4.0 Internacional. 